\title{
Non-parathyroid hypercalcemia associated with paraffin oil injection in 12 younger male bodybuilders: a case series
}

\author{
Anne Sophie Koldkjær Sølling ${ }^{1}$, Birgitte G Tougaard ${ }^{2}$, Torben Harsløf', Bente Langdahl ${ }^{1}$, \\ Helle Kongsbak Brockstedt', Keld-Erik Byg ${ }^{3}$, Per Ivarsen ${ }^{4}$, Ina Karstoft Ystrøm ${ }^{4}$, Frank Holden Mose ${ }^{5}$, \\ Gustaf Lissel Isaksson², Morten Steen Svarer Hansen ${ }^{2,6}$, Subagini Nagarajah²,8, Charlotte Ejersted ${ }^{6}$, \\ Elisabeth Bendstrup ${ }^{7}$ and Lars Rejnmark ${ }^{1}$
}

'Department of Endocrinology and Internal Medicine, THG, Aarhus University Hospital, Aarhus, Denmark, '2Department of Nephrology and Internal Medicine, Regional Hospital Kolding, Kolding, Denmark, ${ }^{3}$ Department of Rheumatology, Odense University Hospital, Odense, Denmark, ${ }^{4}$ Department of Nephrology, Aarhus University Hospital, Aarhus, Denmark, ${ }^{5}$ University Clinic in Nephrology and Hypertension, Regional Hospital West Jutland and Aarhus University, Holstebro, Denmark, ${ }^{6}$ Department of Endocrinology, Odense University Hospital, Odense, Denmark, ${ }^{7}$ Department of Respiratory Diseases and Allergy, Aarhus University Hospital, Aarhus, Denmark, and ${ }^{8}$ Department of Cardiovascular and Renal Research, Institute of Molecular Medicine, University of Southern Denmark, Odense, Denmark

Correspondence should be addressed to A S K Sølling Email

annesoel@rm.dk

\begin{abstract}
Introduction: Injection of paraffin oil to augment muscles size is a troubling phenomenon known to cause a foreign body reaction with formation of granulomas. In a few case reports, long-term side effects have been reported in terms of hypercalcemia and renal failure.

Methods: We identified a case series of 12 male bodybuilders presenting with non-parathyroid hypercalcemia who previously had injected paraffin oil to increase muscles size.

Results: At admission, all patients had moderate-to-severe hypercalcemia with suppressed PTH levels and impaired renal function. Calcitriol levels were within the normal range or slightly elevated. Follow-up measurements showed marked hypercalciuria with nearly normal levels of bone turnover markers. A correlation was found between levels of peptidyl dipeptidase and calcitriol $(R=0.812, P=0.050)$. Treatment with antiresorptive agents seemed less effective than glucocorticoids, which resulted in a significantly lowering of ionized calcium levels and improved renal function, although no patients were cured by this treatment. Immunosuppression with azathioprine or mycophenolate may have a glucocorticoid-saving effect. One patient had surgery with removal of affected muscle tissue, without any apparent effect on plasma calcium levels.

Conclusion: The hypercalcemia and associated hypercalciuria seems to be due to an intestinal hyperabsorption of calcium. It remains to be elucidated, whether an increased calcitriol synthesis within granulomas is the only (main) mechanism by which intestinal calcium absorption is increased. Glucocorticoids seem most appropriate as the first choice for treatment. Bodybuilders should be warned against use of intramuscular oil injections (and other substances), as this may have severe adverse health consequences.
\end{abstract}

(C) 2018 European Society of Endocrinology Printed in Great Britain
European Journal of Endocrinology

(2018) 178, K29-K37
Published by Bioscientifica Ltd. 


\section{Introduction}

Hypercalcemia is a rare condition in young men. When it occurs, hypercalcemia is most often attributable to either primary hyperparathyroidism with high plasma levels of parathyroid hormone (PTH) or malignancies in which PTH levels are supressed. In addition, non-parathyroid hypercalcemia may be caused by other rare conditions such as sarcoidosis, Addison's disease, pheochrormocytoma and intoxication with vitamin A or vitamin D (1). Plasma interleukin-2 receptor and peptidyl-di-peptidase are often measured to diagnose sarcoidosis (2). In recent years, a few case reports have been published on non-parathyroid hypercalcemia attributable to use of various body fillers for cosmetic purposes, including subcutaneous injections with silicone and ruptures of silicone implants, as well as injection of methylmethacrylate and mineral/organic oil $(3,4,5,6,7)$. In three case reports, non-parathyroid hypercalcemia have been reported in bodybuilders who have used intramuscular injections of paraffin oil to augment muscle size $(8,9,10)$. Biopsies from areas injected with body fillers have suggested a foreign body reaction with granuloma formation expressing $1 \alpha$-hydroxylase activity, suggesting that the hypercalcemia is attributable to an increased calcitriol synthesis, which may cause intestinal hyperabsorption of calcium resulting in hypercalcemia. We now report a case series of 12 patients with non-parathyroid hypercalcemia associated with intramuscular injections of paraffin oil.

\section{Methods}

We used a case-based approach by which we from March 2017 to October 2017 identified 12 patients referred to Aarhus University Hospital, Regional Hospital Kolding or Odense University Hospital with non-parathyroid hypercalcemia who all had a history of intramuscular injection with paraffin oil for body contouring. We retrieved data on laboratory findings and results on applied treatment regimes in order to enhance our knowledge on this type of non-parathyroid hypercalcemia. Biochemical analyses were performed by standard methods at hospitals treating the patients. We studied associations between measured indices by calculating Spearman's Rho and assessed effects of treatments by Wilcoxon signed-rank test. Informed consent was obtained from patients for publication of this article.

The Central Denmark Region Committees on Health Research Ethics determined that according to the
Consolidation Act on Research Ethics Review of Health Research Projects, Consolidation Act number 1083 of 15 September 2017 section 14 (1) only health research studies have to be notified to the committees. The committees did not consider the study to be a health research study (section 2 (1)) and therefore the study did not need not be reported to the committees.

\section{Results}

Table 1 shows characteristics of the 12 patients admitted to hospital with moderate-to-severe hypercalcemia. Most of the patients had classical symptoms of hypercalcemia at admission (confusion, lethargy, fatigue, dehydration, gastrointestinal symptoms and symptoms from the musculoskeletal system). All were males with a mean age of 34 (range: 29-40) years and a mean BMI of $28.8 \mathrm{~kg} /$ $\mathrm{m}^{2}$. All had a history of using paraffin oil injections and were admitted on average five (range: $1-8$ ) years after the injections. In seven patients, data on the amount of the injected paraffin oil were available; ranging from 600 to $2000 \mathrm{~mL}$. All patients had used the injections for cosmetic purposes in order to augment apparent muscle size. All of the men were former bodybuilders who also reported abuse of other substances like anabolic steroids, testosterone, growth hormone, T3-hormone and amphetamine. In addition, most of the men also reported use of numerous vitamins, dietary supplements, energy drinks and proteincreatine powder on a daily basis.

\section{Biochemical findings at the time of admission}

Table 2 shows biochemical findings at the time of admission and prior to any treatment. Plasma ionized calcium $\left(\mathrm{P}_{-}-\mathrm{Ca}^{2+}\right)$ levels were in the range of $1.37-2.03$ (mean: 1.75, 95\% CI: 1.62-1.87) mmol/L. Plasma PTH levels were suppressed with values below the lover limit of the reference interval in ten of the 12 patients. Plasma phosphate levels (mean: 1.05; 95\% CI: $0.86-1.23 \mathrm{mmol} / \mathrm{L}$ ) were within the reference interval for all cases, except for one patient who had slight hyperphosphatemia. Eleven of the patients had impaired renal function (mean eGFR: 35 , 95\% CI: $25-46 \mathrm{~mL} / \mathrm{min}$ ) with an eGFR $<60 \mathrm{~mL} /$ min and $42 \%$ had an eGFR $\leq 30 \mathrm{~mL} / \mathrm{min}$. Mean levels of 25-hydroxyvitamin D (25OHD) were 50 (95\% CI: 39-61) $\mathrm{nmol} / \mathrm{L}$ and none of the patients had levels suggestive of vitamin D intoxication. Moreover, plasma calcitriol levels were low to normal for all eight patients who had this measurement performed at admission. Plasma 
interleukin-2 receptor levels were elevated in all patients $(n=10)$ and levels of peptidyl-di-peptidase were elevated in nine of the 11 patients who had this measurement performed (Table 1).

\section{Biochemical measurement during follow-up}

Plasma levels of 25OHD and calcitriol were re-measured in all patients, showing findings similar to initial measurements with no signs of vitamin D intoxication. Plasma interleukin-2 receptor was also re-measured showing a significant decline $(P=0.028)$ from a mean value of $1202(747-1656) \mathrm{kU} / \mathrm{I}$ to 700 (432-968) $\mathrm{kU} / \mathrm{I}$. 24-h urinary calcium was measured in eight patients, among whom seven had elevated levels. Vitamin A levels were above the upper limit of the reference interval in five of the 11 patients who had this measurement performed. Plasma levels of parathyroid hormone-related peptide (PTHrP) were below the lower limit of detection in the three patients who had this measurement performed. eGFR correlated inversely with plasma levels of ionized calcium $(R=-0.643, P=0.024)$ and phosphate $(R=-0.613$, $P=0.034)$, whereas there was a positive correlation between plasma levels of peptidyl di-peptidase and calcitriol $(R=0.812, P=0.050)$.

\section{Imaging}

A positron emission tomography with ${ }^{18}$ F-fluorodeoxyglucose with integrated computed tomography $\left({ }^{18} \mathrm{~F}-\mathrm{FDG}\right.$ PET/CT) scan of the thorax, abdomen and pelvis was performed in nine of the patients. In general, the scans showed heavily increased FDG uptake in the muscles, where the patients had injected paraffin oil (Fig. 1). A dual-energy x-ray absorptiometry (DXA) scan was performed in five of the patients, showing a normal bone mineral density at all sites. On CT scans, kidney stones and/or nephrocalcinosis was found in six patients.

\section{Effects of treatment with glucocorticoids}

Treatment with glucocorticoids in a daily dose $\geq 25 \mathrm{mg}$ of prednisolone was administered to 11 of the patients for one month and was continued for two months in nine patients and for six months in six of the patients. Immediately prior to initiation of therapy, levels of $\mathrm{Ca}^{2+}$ were 1.70 (95\% CI: 1.60-1.80) $\mathrm{mmol} / \mathrm{L}$, which on average decreased to $1.40(1.31-1.49) \mathrm{mmol} / \mathrm{L}$ after one month of therapy $(P=0.006)$ to $1.38(1.30-1.45) \mathrm{mmol} / \mathrm{L}$ after two 


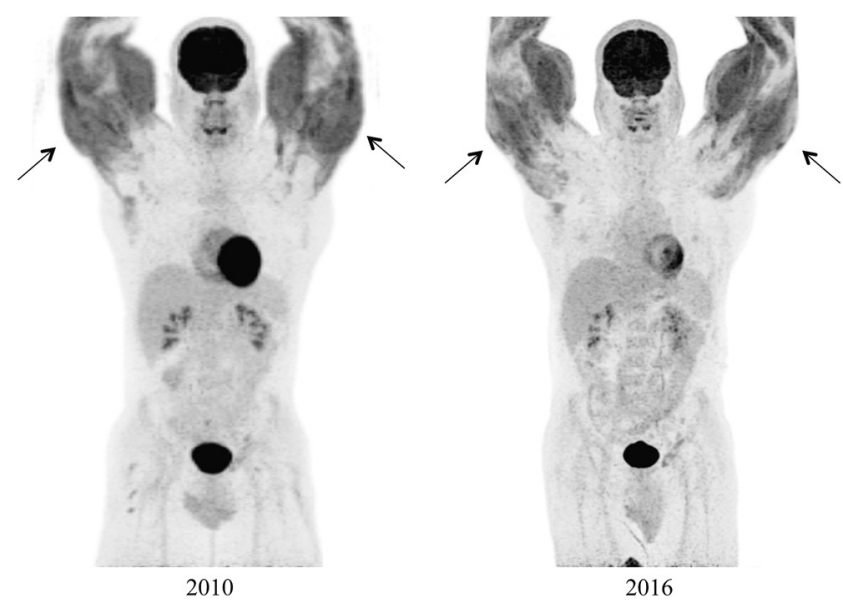

Figure 1

18F-FDG PET/CT from 2010 (left) shows heavily increased FDG uptake (arrows) in the upper arms. The scan was repeated in 2016 (right) to assess signs of progression; however, the scans were identical. Both scans also shows normal increased physiological uptake in the cerebrum, heart and urinary system.

months of therapy $(P=0.008)$ and to $1.42(1.29-1.54)$ $\mathrm{mmol} / \mathrm{L}$ after 6 months of therapy $(P=0.028)$ (Fig. 2). Concomitantly, renal function improved significantly during the first two months of treatment with prednisolone (Fig. 2). The mean eGFR increased from 35 (25-45) $\mathrm{mL} / \mathrm{min}$ to $46(36-57) \mathrm{mL} / \mathrm{min}$ after one month $(P=0.016)$ and to $49(37-63) \mathrm{mL} / \mathrm{min}$ after two months $(P=0.012)$. The current daily dose of prednisolone did not correlate with the actual measurement of $\mathrm{p}-\mathrm{Ca}^{2+}(r=0.489$, $P=0.11$ ). One of the patients received treatment with $100 \mathrm{mg}$ hydrocortisone four times daily during admission in 2007, which led to a decline in $\mathrm{p}-\mathrm{Ca}^{2+}$ from 1.87 to $1.34 \mathrm{mmol} / \mathrm{L}$. However, the prednisolone dosage could not be reduced below $37.5 \mathrm{mg}$ prednisolone daily without rapid increases in $\mathrm{p}-\mathrm{Ca}^{2+}$ and worsening of the kidney function. However, no apparent effect of prednisolone therapy was evident in three of the patients. In order to exclude lack of compliance, one of these patients was treated with hydrocortisone intravenously in a dose of $500 \mathrm{mg}$ per week for 12 weeks. However, this did not have marked effects on $\mathrm{p}-\mathrm{Ca}^{2+}$, which after the end of the highdose treatment was $1.45 \mathrm{mmol} / \mathrm{L}$.

\section{Effects of treatment with antiresorptive agents}

In June 2015, December 2015 and April 2016, case \#1 was treated with $5 \mathrm{mg}$ zoledronic acid followed by denosumab 60 mg twice in June 2016 and 120 mg denosumab in August 


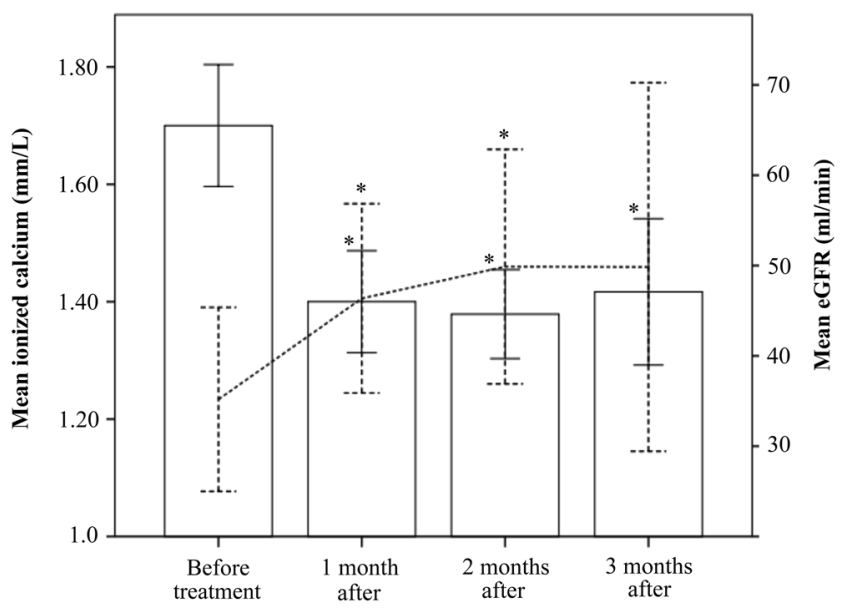

Figure 2

Effects of treatment with prednisolone in a dose of $\geq 25 \mathrm{mg} /$ day on eGFR (dotted line) and plasma levels of ionized calcium (columns). Mean with $95 \%$ confidence intervals. *Significantly different from measurement before treatment.

2016. However, $\mathrm{Ca}^{2+}$ remained elevated $>1.6 \mathrm{mmol} / \mathrm{L}$. Similar, treatment with prednisolone $50 \mathrm{mg} /$ day and denosumab $60 \mathrm{mg}$ twice during 2017 did not have any lasting effect on $\mathrm{P}-\mathrm{Ca}^{2+}$ in case \#6. On the other hand, a decline in $\mathrm{P}_{-} \mathrm{Ca}^{2+}$ from $1.63 \mathrm{mmol} / \mathrm{L}$ to $1.43 \mathrm{mmol} / \mathrm{L}$ was seen in case \#2 after treatment with $5 \mathrm{mg}$ zoledronic acid in November 2015 and May 2016.

Case \#3 received treatment with glucocorticoid (doserange $12.5-25 \mathrm{mg}$ ) and pamidronic acid $60 \mathrm{mg}$ twice in June 2016 and July 2016 as well as $60 \mathrm{mg}$ of denosumab in August 2016. Despite treatment, calcium levels remained elevated 1.47-2.01 mmol/L (years 2016-2017). Surprisingly, during admission in September 2017, $\mathrm{P}-\mathrm{Ca}^{2+}$ levels dropped from $1.57 \mathrm{mmol} / \mathrm{L}$ to $1.30 \mathrm{mmol} / \mathrm{L}$ in only two days. The patient admitted to have been taking performance-enhancing drugs throughout the last couple of months.

After treatment with $30 \mathrm{mg}$ of pamidronate acid in case \#9, $\mathrm{P}-\mathrm{Ca}^{2+}$ dropped from 1.73 to $1.5 \mathrm{mmol} / \mathrm{L}$ in only two days and stayed within the normal range during the following months. Similarly, case \#7 became normocalcemic after two infusions of $30 \mathrm{mg}$ pamidronate acid, but hypercalcemia recurred after a few weeks. Case \#4 also became normocalcemic after treatment with $30 \mathrm{mg}$ of pamidronate acid in combination with prednisolone (daily dose of 10-40 mg). However, hypercalcemia recurred after only three months, and despite treatment with an additional dose of pamidronate acid $30 \mathrm{mg}$ and prednisolone (daily dose of $5-25 \mathrm{mg}$ ), $\mathrm{P}-\mathrm{Ca}^{2+}$ continued to increase and reached a level of $1.99 \mathrm{mmol} / \mathrm{L}$. The patient received further three infusions with pamidronate acid (60, 90 and $60 \mathrm{mg}$, respectively) with only four days apart, which was followed by a decrease in $\mathrm{P}-\mathrm{Ca}^{+2}$ to $1.38 \mathrm{mmol} / \mathrm{L}$.

In case \#5, following treatment with pamidronate $50 \mathrm{mg}$ and Solu-Medrol $80 \mathrm{mg}$ in August 2014, $\mathrm{P}-\mathrm{Ca}^{2+}$ dropped from 1.80 to $1.42 \mathrm{mmol} / \mathrm{L}$. During the following year, the patient received additional treatment with azathioprine and prednisolone. Four additional infusions of $30 \mathrm{mg}$ of pamidronate acid and azathioprine $150 \mathrm{mg}$ was given in 2015-2016. However, this did not result in normocalcemia.

\section{Other treatments regimes}

Treatment with azathioprine, hydroxychloroquine, mycophenolate or infliximab was tried in a few patients in addition to prednisolone and antiresorptive agents (Table 3). Treatment with azathioprine in 5 patients (or mycophenolate in two patients) was after three months

Table 3 Treatment regimens.

\begin{tabular}{|c|c|c|c|c|c|c|c|c|c|c|c|c|}
\hline & \multicolumn{12}{|c|}{ Cases } \\
\hline & 1 & 2 & 3 & 4 & 5 & 6 & 7 & 8 & 9 & 10 & 11 & 12 \\
\hline \multicolumn{13}{|l|}{ Treatment with } \\
\hline Glucocorticoids & + & + & + & + & + & + & + & + & & + & + & + \\
\hline Denosumab & + & & + & & & + & & & & & & \\
\hline Zoledronic acid & + & + & & & & & & & & & & \\
\hline Pamidronic acid & + & & + & + & + & & + & & + & & & \\
\hline Miacalcic & & & & & & & & & & + & & \\
\hline Azathioprine & + & & & & + & + & + & & & & + & + \\
\hline Plaquinil & & & & & + & & & & & & & \\
\hline Mycophenolate & & & & & + & & & & & & + & \\
\hline Infliximab & + & & & & & & & & & & & \\
\hline $\begin{array}{l}\text { Surgical removal the } \\
\text { paraffinomas }\end{array}$ & + & & & & & & & & & & & \\
\hline
\end{tabular}


associated with a $14 \%$ (range 0-25\%) mean decrease in creatinine, $8 \%$ (range 0-18\%) in $\mathrm{Ca}^{2+}$ and 18\% (range 0-60\%) reduction in prednisolone dose. All patients had response in at least one of the parameters. Treatment with fluids was included in all of the mentioned treatment regimes (antiresorptive agents, glucocorticoids and others).

\section{Effect of surgery}

Case \#1 had surgery done in October 2016. The tissue on both arms, in which he had injected paraffin oil, was surgically removed. As the operation was performed abroad, the description of the exact procedure is not available. However, according to the patient, the surgeon removed approximately $2.2 \mathrm{~kg}$ of tissue from his arms. Despite the operation, the hypercalcemia persisted with a $\mathrm{P}-\mathrm{Ca}^{2+}$ level of 1.66 (reference range: $1.18-1.32$ ) $\mathrm{mmol} / \mathrm{L}$ in June 2017.

\section{Bone turn over markers}

Plasma levels of alkaline phosphatase (ALP) are considered as a marker of bone formation. At admission to hospital and prior to initiation of any antiresorptive therapy, ALP levels were within the reference interval in all patients with a mean (95\% CI) of 70 (55-85) U/L (Table 2). During follow-up, ALP levels remained within the reference interval. As measured at a random time during follow-up and after initiation of the different therapies, most patients had normal levels of the bone formative marker procollagen type I N propeptide (PINP), as well as normal levels of C-terminal telopeptide (CTx), which reflects bone resorption.

\section{Discussion}

In the present paper, we report findings on 12 males admitted to our hospitals with non-parathyroid hypercalcemia, which was characterized by elevated levels of peptidyl-di-peptidase and interleukin-2 receptor, lowto-normal levels of calcitriol and bone turnover markers as well as hypercalciuria. All patients had a history of intramuscular injections with paraffin oil used for increasing apparent muscle size.

Historically, paraffin (mineral) oil has been used for correcting disfigurements of the face or other parts of the body since the early 20th century, but the use was discontinued due to severe side effects. Short-term complications associated with injection of paraffin oil include inflammation, ulceration and skin necrosis (11). Potential long-term adverse effects to injections with paraffin oil was reported by Albitar et al. in a 63-year-old man who had paraffinomas associated with severe hypercalcemia, renal failure, oil pneumonia and granulomatous skin lesions (10). Gyldenløve et al. found severe hypercalcemia $\left(\mathrm{P}-\mathrm{Ca}^{2+} 2.29 \mathrm{mmol} / \mathrm{L}\right)$ with low levels of PTH and 25-hydroxyvitamin D, renal insufficiency, nephrocalcinosis and elevated levels of angiotensinconverting enzyme, interleukin receptor and calcitriol in a 23-year-old bodybuilder who had injected himself with paraffin oil (8). Similar findings have been reported in a few additional case reports of patients who have used oil injections (paraffin oil, unspecified mineral oil, baby oil) for body contouring $(9,12,13,14)$.

It has been suggested, that paraffin oil induces a foreign body reaction, which results in formation of granulomas, known as paraffinomas (10). Immunohistochemical staining have demonstrated a high expression of CYP27B1 in biopsies of paraffinomas $(8,12)$. This may suggest that the hypercalcemia could be driven by a high extra-renal 1-alpha-hydroxylase activity leading to an abnormal high conversion of 25-hydroxyvitamin D into calcitriol, similar to a state of vitamin D intoxication. Our findings of lownormal levels of biochemical markers of bone turnover do not support an increased efflux of calcium from bone as the cause of hypercalcemia. The hypercalcemia and accompanying hypercalciuria might be due to an intestinal hyperabsorption of calcium. However, it is worth noticing that most of our cases actually had plasma calcitriol levels within the normal range. Perhaps a small increase within the normal reference range of calcitriol from a specific 'set point' of the individual patients is sufficient to stimulate the increases in calcium. Interestingly, our analyses showed a significant association between plasma levels of calcitriol and peptidyl di-peptidase, whereas the degree of hypercalcemia did not correlate with levels of calcitriol. The causal importance of calcitriol in the development of hypercalcemia may be further questioned by our findings of normal plasma levels of phosphate. In patients with vitamin D intoxication, plasma phosphate levels are typically elevated due to an increased intestinal absorption. Future studies should aim to evaluate phosphate homeostasis within this group of patients in a more precise manner, including renal phosphate excretion with calculation of the tubular maximum reabsorption of phosphate (TmP) to GFR (TmP/GRF) and levels of fibroblast growth factor 23. Additional studies to be performed in order to elucidate mechanisms involved 
in the development of hypercalcemia may include potential effects of different cytokines on intestinal calcium absorption through vitamin D independent pathways. Furthermore, within our group of patients, as well as reported in previous case reports $(9,14)$, multisubstance abuse is not uncommon, including abuse of anabolic steroids, growth hormone, testosterone and erythropoietin. For example, treatment with growth hormone has been shown to cause hypercalcemia through a vitamin D independent mechanism (15). A drug screening for anabolic steroids and other performanceenhancing drugs would have been preferable. We cannot exclude that abuse of such substances may have contributed to the hypercalcemia.

Our study showed a significant lowering of $\mathrm{P}-\mathrm{Ca}^{2+}$ levels in response to treatment with glucocorticoids, whereas effects of treatment with antiresorptive agents were more modest (or lacking), supporting a too high intestinal calcium absorption as the mechanism of action for the hypercalcemia. However, despite a decrease in $\mathrm{P}-\mathrm{Ca}^{2+}$ levels, most patients did not achieve normocalcemia in response to therapy and the response was only modest (or lacking) in some patients. We cannot exclude that this (at least in part) may be explained by lack of compliance or treatment with too low doses. Mean eGFR also improved significantly in response to treatment with glucocorticoids. This suggests that the impaired renal function was due to mainly reversible causes, which may be due to several mechanisms including an impaired concentrating ability of the distal tubule causing polyuria with dehydration, as well as direct alterations of intravascular tone, and glomerular permeability (16). Most patients required sustained moderate dose of glucocorticoid treatment, which is very worrying in a long-time perspective due to a high risk of glucocorticoidinduced side effects. Antiresorptive drugs as well as immunosuppression with azathioprine or mycophenolate seems to have a general minor additive effect and in some patients a glucocorticoid-saving effect.

Many of the patients underwent extensive diagnostic evaluation for non-parathyroid hypercalcemia, as vitamin $\mathrm{D}$ intoxication was not considered due to normal levels of 25OHD and calcitriol. A diagnosis of sarcoidosis was presumed in several of the patients as elevated levels of peptidyl-dipeptidase and soluble interleukin 2 receptor are considered as an index for disease activity in sarcoidosis (17). In sarcoidosis, several studies have reported a significant correlation between plasma levels $\mathrm{Ca}^{2+}$ and peptidyl-dipeptidase, whereas no correlations have been reported between levels of calcitriol and peptidyl- dipeptidase $(18,19)$. In contrast to these findings, our analyses showed a significant correlation between levels of peptidyl-dipeptidase and calcitriol. To the best of our knowledge, a direct pathophysiological relation between levels of calcitriol/ $\mathrm{Ca}^{2+}$ and peptidyl-dipeptidase/soluble interleukin 2 receptor has not been reported. Most likely, elevated levels of peptidyl-dipeptidase and IL-2 receptor reflect a foreign body reaction in terms of the granulomatous process, including the immune response with activation of T-lymphocytes resulting in increased levels of soluble interleukin receptor 2 (15).

Elevated vitamin $\mathrm{A}$ is a well-known rare cause of hypercalcemia (20). Studies have suggested, that the hypercalcemia might be caused by increased bone resorption mediated directly by vitamin A $(20,21)$. However, inhibition of boneresorption by bisphosphonates or denosumab did not lower calcium levels to any major degree, suggesting that vitamin A was not the cause of hypercalcemia in our patients. Five of 11 (45\%) of our patients had vitamin A levels above the upper limit of the reference interval, and we can of course not exclude that this may have contributed to the hypercalcemia. However, it seems unlikely, that hypervitaminosis $\mathrm{A}$ is the sole explanation of the hypercalcemia in these patients.

The timespan from paraffin injection until the formation of paraffinomas has been reported to be very variable (10), which is supported by our findings. The total load of paraffin oil could only be recorded in five patients and did not correlate with the degree of hypercalcemia at time of admission. However, the amount injected could not be verified and the estimate is probably associated with some uncertainties.

Surgical removal of paraffinomas and the effect hereof is only sparsely documented. Ikander et al. described four patients who had surgery done, but effects on biochemical indices were not reported in this case series (22). In our patient who had large amounts of affected tissue removed surgically, the hypercalcemia persisted, suggesting that this treatment approach is not unconditional applicable.

Our paper has several limitations. Data reported have not been collected in a protocolled manner, but result from measurements performed and treatments applied by the discretion of different physicians responsible for the treatment of the patients. Furthermore, biochemical results reported have been analyzed on different laboratories, using different assays. Additionally, the 1-alpha hydroxylase expression in the granulomas has not been confirmed in biopsies from the patients, which would have been preferable since there could be other explanations for the hypercalcemia like multi-substance 
abuse. As mentioned, it has previously been shown with immunohistochemical staining, that biopsies of paraffinomas have demonstrated a high expression of CYP27B1 $(8,12)$ and we speculate, that similar findings may apply to our patients. Of importance, our study is observational and do not allow for conclusions on the pathogenesis of hypercalcemia.

Furthermore, the evaluation of findings from the $18 \mathrm{~F}$ FDG PET/CT scans was based on visual inspection, as we did not have access to a quantitative evaluation. Finally, investigation of intestinal calcium absorption has not been possible in our patients.

However, despite these important limitations, our case series is, to the best of our knowledge, the largest collection of patients who have developed severe adverse effects to intramuscular injections with paraffin oil. No cure seems to exist and patients seem to have a poor prognosis, as long-term treatment with high doses of glucocorticoids is needed to avoid severe hypercalcemia with deterioration of renal function.

\section{Conclusion}

Non-parathyroid hypercalcemia may be attributable to intramuscular injection with paraffin oil. The cause of hypercalcemia seems to be increased intestinal calcium absorption accompanied by hypercalciuria. Our data do not uniformly support an increased synthesis of calcitriol as the only (main) mechanism by which calcium absorption is increased. While waiting for follow-up results on patients treated with inhibitors of 1- alpha hydroxylase like hydroxychloroquine, treatment with glucocorticoids should be first treatment choice although patients may not achieve normocalcemia in response to therapy. It seems reasonable to recommend a diet low in calcium. Effects of treatment with antiresorptive agents are probably only very modest. Bodybuilders should be warned against intramuscular injections with paraffin oil (and other substances), as no cure exists if hypercalcemia develops.

\section{Declaration of interest}

Helle Kongsbak Brockstedt has been a member of Amgen Advisory Board. Lars Rejnmark has received lecture fees from Amgen and Eli Lilly and has consulted for NPS Pharma, Shire and Alexion. Torben Harsløf received lecture fees from Amgen, Astra Zeneca, and Eli Lilly. Bente Langdahl is a consultant for MSD, Amgen, Eli Lilly, and UCB and has received lecture fees from MSD, Eli Lilly, and Amgen. All other authors have nothing to declare.

\section{Funding}

This research did not receive any specific grant from any funding agency in the public, commercial or not-for-profit sector.

\section{References}

1 Jacobs TP \& Bilezikian JP. Rare causes of hypercalcemia. Journal of Clinical Endocrinology and Metabolism 200590 6316-6322. (https:// doi.org/10.1210/jc.2005-0675)

2 Valeyre D, Prasse A, Nunes H, Uzunhan Y, Brillet PY \& MüllerQuernheim J. Sarcoidosis. Lancet 2014383 1155-1167. (https://doi. org/10.1016/S0140-6736(13)60680-7)

3 Hindi SM, Wang Y, Jones KD, Nussbaum JC, Chang Y, Masharani U, Bikle D, Shoback DM \& Hsiao EC. A case of hypercalcemia and overexpression of CYP27B1 in skeletal muscle lesions in a patient with HIV infection after cosmetic injections with polymethylmethacrylate (PMMA) for wasting. Calcified Tissue International 201597 634-639. (https://doi.org/10.1007/s00223-0150048-8)

4 Negri AL, Rosa Diez G, Del Valle E, Piulats E, Greloni G, Quevedo A, Varela F, Diehl M \& Bevione P. Hypercalcemia secondary to granulomatous disease caused by the injection of methacrylate: a case series. Clinical Cases in Mineral and Bone Metabolism 201411 44-48.

5 Rados DV \& Furlanetto TW. An unexpected cause of severe and refractory PTH-independent hypercalcemia: case report and literature review. Archives of Endocrinology and Metabolism 201559 277-280. (https://doi.org/10.1590/2359-3997000000051)

6 Melnick S, Abaroa-Salvatierra A, Deshmukh M \& Patel A. Calcitriol mediated hypercalcaemia with silicone granulomas due to cosmetic injection. BMJ Case Reports 20162016 bcr2016217269. (https://doi. org/10.1136/bcr-2016-217269)

7 Agrawal N, Altiner S, Mezitis NHE \& Helbig S. Silicone-induced granuloma after injection for cosmetic purposes: a rare entity of calcitriol-mediated hypercalcemia. Case Reports in Medicine 2013 2013 807292. (https://doi.org/10.1155/2013/807292)

8 Gyldenløve M, Rørvig S, Skov L \& Hansen D. Severe hypercalcaemia, nephrocalcinosis, and multiple paraffinomas caused by paraffin oil injections in a young bodybuilder. Lancet 2014383 2098. (htps://doi. org/10.1016/S0140-6736(14)60806-0)

9 Schäfer CN, Guldager H \& Jørgensen HL. Multi-organ dysfunction in bodybuilding possibly caused by prolonged hypercalcemia due to multi-substance abuse: case report and review of literature. International Journal of Sports Medicine 201132 60-65. (https://doi. org/10.1055/s-0030-1267200)

10 Albitar S, Genin R, Fen-Chong M, Schohn D, Riviere JP, Serveaux MO Chuet C \& Bourgeon B. Multisystem granulomatous injuries 28 years after paraffin injections. Nephrology Dialysis Transplantation 199712 1974-1976. (https://doi.org/10.1093/ndt/12.9.1974)

11 Henriksen TF, Løvenwald JB \& Matzen SH. Paraffin oil injection in bodybuilders calls for preventive action. Ugeskrift for Laeger 2010172 219-220.

12 Moraitis AG, Hewison M, Collins M, Anaya C \& Holick MF. Hypercalcemia associated with mineral oil-induced sclerosing paraffinomas. Endocrine Practice 201319 e50-e56. (https://doi. org/10.4158/EP12092.CR)

13 El Muayed M, Costas AA \& Pick AJ. 1,25-Dihydroxyvitamin D-mediated hypercalcemia in oleogranulomatous mastitis (paraffinoma), ameliorated by glucocorticoid administration. Endocrine Practice 201016 102-106. (https://doi.org/10.4158/ EP09008.CRR)

14 Bento C, Velho P \& Carvalho M. Lots of steroids and vitamins, tons of complications. Hypercalcemia and nephrocalcinosis as important 
complications of performance-enhancing drugs. Nefrología 201535 598-600. (https://doi.org/10.1016/j.nefro.2015.09.005)

15 Rubin LA \& Nelson DL. The soluble interleukin-2 receptor: biology, function, and clinical application. Annals of Internal Medicine 1990 113 619-627. (https://doi.org/10.7326/0003-4819-113-8-619)

16 Moysés-Neto M, Guimarães FM, Ayoub FH, Vieira-Neto OM, Abrão J, Costa C \& Dantas M. Acute renal failure and hypercalcemia. Renal Failure 200628 153-159.

17 Keijsers RG, Verzijlbergen FJ, Oyen WJ, van den Bosch JM, Ruven HJ, van Velzen-Blad H \& Grutters JC. 18F-FDG PET, genotype-corrected ACE and SIL-2R in newly diagnosed sarcoidosis. European Journal of Nuclear Medicine and Molecular Imaging 200936 1131-1137. (https:// doi.org/10.1007/s00259-009-1097-x)

18 Hamada K, Nagai S, Tsutsumi T \& Izumi T. Ionized calcium and 1,25-dihydroxyvitamin D concentration in serum of patients with sarcoidosis. European Respiratory Journal 199811 1015-1020. (https:// doi.org/10.1183/09031936.98.11051015)
19 Lieberman J, Nosal A, Schlessner A \& Sastre-Foken A. Serum angiotensin-converting enzyme for diagnosis and therapeutic evaluation of sarcoidosis. American Review of Respiratory Disease 1979120 329-335. (https://doi.org/10.1164/ arrd.1979.120.2.329)

20 Conaway HH, Henning P \& Lerner UH. Vitamin A metabolism, action, and role in skeletal homeostasis. Endocrine Reviews 201334 766-797. (https://doi.org/10.1210/er.2012-1071)

21 Conaway HH, Pirhayati A, Persson E, Pettersson U, Svensson O, Lindholm C, Henning P, Tuckermann J \& Lerner UH. Retinoids stimulate periosteal bone resorption by enhancing the protein RANKL, a response inhibited by monomeric glucocorticoid receptor. Journal of Biological Chemistry 2011286 31425-31436. (https://doi. org/10.1074/jbc.M111.247734)

22 Ikander P, Nielsen AM \& Sørensen JA. Injection of synthol in a bodybuilder can cause chronic wounds and ulceration. Ugeskrift for Laeger 2015177.

Received 18 January 2018

Revised version received 19 March 2018

Accepted 29 March 2018 